\title{
Effect of surface losses on soliton propagation in Josephson junctions
}

\author{
Davidson, A.; Pedersen, Niels Falsig; Pagano, S.
}

\section{Published in:}

Applied Physics Letters

Link to article, DOI:

10.1063/1.96961

Publication date:

1986

Document Version

Publisher's PDF, also known as Version of record

Link back to DTU Orbit

Citation (APA):

Davidson, A., Pedersen, N. F., \& Pagano, S. (1986). Effect of surface losses on soliton propagation in Josephson junctions. Applied Physics Letters, 48(19), 1306-1308. https://doi.org/10.1063/1.96961

\section{General rights}

Copyright and moral rights for the publications made accessible in the public portal are retained by the authors and/or other copyright owners and it is a condition of accessing publications that users recognise and abide by the legal requirements associated with these rights.

- Users may download and print one copy of any publication from the public portal for the purpose of private study or research.

- You may not further distribute the material or use it for any profit-making activity or commercial gain

- You may freely distribute the URL identifying the publication in the public portal

If you believe that this document breaches copyright please contact us providing details, and we will remove access to the work immediately and investigate your claim 


\title{
Ethect of surtace losses on solliton propagation hit Josephson lumctions
}

\author{
A. Davidson and N. F. Pedersen ${ }^{\text {a) }}$ \\ IBM Research, Yorktown Heights, New York 10598
}

\author{
S. Pagano \\ The Technical University of Denmark, Laboratory for Applied Mathematical Physics, DK2800, Lyngby, \\ Denmark
}

(Received 6 February 1986; accepted for publication 17 March 1986)

\begin{abstract}
We have explored numerically the effects on soliton propagation of a third order damping term in the modified sine-Gordon equation. In Josephson tunnel junctions such a term corresponds physically to quasiparticle losses within the metal electrodes of the junction. We find that this loss term plays the dominant role in determining the shape and stability of the soliton at high velocity.
\end{abstract}

Soliton propagation in Josephson Junctions has been considered for many practical applications such as microwave oscillators, ${ }^{1}$ logic circuits, ${ }^{2}$ and analog amplification. ${ }^{3}$ In most cases the theoretical models include only the effect of damping due to tunneling of quasiparticles. In a recent paper ${ }^{4}$ it was demonstrated experimentally that the effect of surface impedance damping may be quite large and even dominate the soliton propagation in some cases. In this letter we investigate numerically soliton propagation in annular Josephson junctions in the presence of realistic surface impedance damping. We find that the surface impedance losses introduce not only quantitative changes but also new qualitative features.

Soliton motion in long one-dimensional Josephson junctions is approximately described by the modified sine-Gordon equations ${ }^{5}$

$$
-\phi_{x x}+\phi_{t}+\sin \phi=-\alpha \phi_{1}+\beta \phi_{x x t}+\eta .
$$

In this equation the time $t$ is normalized to the inverse of the plasma frequency $1 / \omega_{0}$, and the spatial variable $x$ is normalized to the Josephson penetration depth $\lambda_{j}$. We assume a uniform bias current $\eta$, which is normalized to the total critical current of the junction. Velocity is normalized to the velocity of light in the barrier, $\bar{c}=\omega_{0} \lambda_{j}$. In these units the voltage $V=\phi_{t}$ is normalized to $\omega_{0} h / 4 \pi e$ and for a junction of normalized length $l$ is related to the velocity $u$ of a single soliton by $V=2 \pi u / l$.

In Eq. (1) two damping mechanisms are present: the quasiparticle tunneling loss is set by the coefficient $\alpha$ and the surface loss in the electrodes is set by the coefficient $\beta$. In experiments these coefficients are typically between 0.001 and 0.3 depending on the junction material and the temperature.

We assume periodic boundary conditions to Eq. (1) with one soliton, i.e., $\phi(0, t)=\phi(l, t)+2 \pi$. Throughout the simulations we have used a line length of $l=8$. Our numerical techniques are discussed in an earlier publication. ${ }^{6}$ However, the surface loss term demanded finer spatial and temporal grids than were used previously, as low as 0.02 spatially and 0.005 for the time.

\footnotetext{
- 'Permanent address: Physics Laboratory I, Technical University of Denmark, DK2800, Lyngby, Denmark.
}

The major qualitative features of this system may be understood from the pure sine-Gordon equation [corresponding to $\eta=\alpha=\beta=0$ in Eq. (1) ] and results from perturbation theory. In perturbation theory $y^{5.4}$ the soliton of Eq. (1) obtains a steady-state velocity given by

$$
\eta=\frac{4}{\pi} u \gamma(u)\left(\alpha+\frac{\beta}{3} \gamma^{2}(u)\right)
$$

if the length of the line, $l$, is sufficiently large. Here $\gamma(u)$ is the Lorentz factor given by $\gamma(u)=\left(1-u^{2}\right)^{-0.5}$. If $\beta=0$ the result for the voltage, $V$, is particularly simple:

$$
V=\frac{2 \pi u}{l}=\frac{2 \pi / l}{\sqrt{1+(4 \alpha / \pi \eta)^{2}}}
$$

As the velocity is increased by the application of a bias current $\gamma(u)$ increases, and a Lorentz contraction occurs; the width of the soliton is given approximately by $1 / \gamma(u)$. The maximum voltage of the soliton may then be expressed as $2 u \gamma(u)$. As $u \rightarrow 1$ however, deviations from perturbation theory are expected to occur. ${ }^{7}$ The phenomenon of Lorentz contraction of the soliton may be observed from Fig. 1, which shows for different parameter values the absolute value of the soliton voltage in a logarithmic plot. In Fig. I (a) the bias current is low and hence $\gamma(u)$ is close to $k$. The line shape is close to that of a sine-Gordon soliton given by ${ }^{5}$

$$
\phi_{t}^{\mathrm{SG}}=4 u \gamma(u) e^{\xi} /\left(1+e^{2 \xi}\right), \quad \xi=(x-u t) \gamma(u) .
$$

Figures $1(\mathrm{~b})$ and 1 (c) show the results for a high value

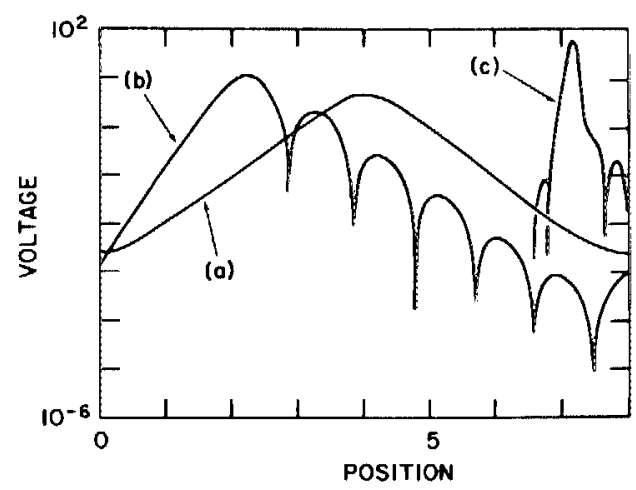

FIG. I. Soliton line shapes. (a) $\alpha=0.02, \beta=0.01, \eta=0.1$; (b) $\alpha=0.02$, $\beta=0.01, \eta=0.675$; (c) $\alpha=0.02333, \beta=0, \eta=0.675$. Numerical value of the voltage is shown. 
of the bias current, $\eta=0.675$ with and without $\beta$ damping. The parameters are chosen such that the low bias behavior is the same for the two curves. In Fig. 1(c) we see that for $\beta=0, \alpha<1$, a substantial Lorentz contraction occurs. With $\beta \neq 0$ [Fig. 1(b)] we note that in contradiction to perturbation theory, no Lorentz contraction has occurred, and that oscillations have appeared on the trailing edge of the soliton. Although not visible from the type of plot used here (absolute value) the voltage goes negative at the $2 \mathrm{nd}$, 4 th, and 6 th maximum in Fig. 1(b). The oscillations in voltage correspond to a decaying oscillatory overshoot in phase.

We have verified that the oscillations in Fig. 1(b) are real and do not depend on the spatial grid used in the simulation. For the case of pure $\alpha$ damping exponentially diminishing oscillations have been found trailing the soliton in earlier numerical simulations. ${ }^{8}$ Although we did observe two small sidelobes attached to the soliton, as in Fig. 1(c), we did not observe any extended, damped oscillations when the spatial grid in the simulation was made small enough, for the case without $\beta$ loss.

For the case when the $\beta$ loss dominates, the spatial oscillations at the trailing edge of the soliton may eventually cause the junction to switch by introducing an additional soliton-antisoliton pair. This is illustrated in Fig. 2 which shows computer-generated pictures of the equivalent pendulum array ${ }^{5}$ for three different values of the bias current. Figure 2(a) with $\eta=0.3$ shows the single soliton on the line when the phase overshoot has not yet developed. Figure 2(b) shows a stable solution with phase overshoot (also shown directly in the superimposed solution for the phase) at $\eta=0.675$. Figure 2 (c) at $\eta=0.71$ shows a snapshot (unstable) at a time just before the overshoot creates an additional soliton-antisoliton pair. This spontaneous creation of more solitons makes the entire junction unstable causing it to switch. We note further that when the junction switches, $u \rightarrow 1$, as will be demonstrated below.

Figure 3 shows in more detail the behavior of the junction with surface impedance damping as the bias current is increased. Figure 3(a) shows the slopes of the leading and

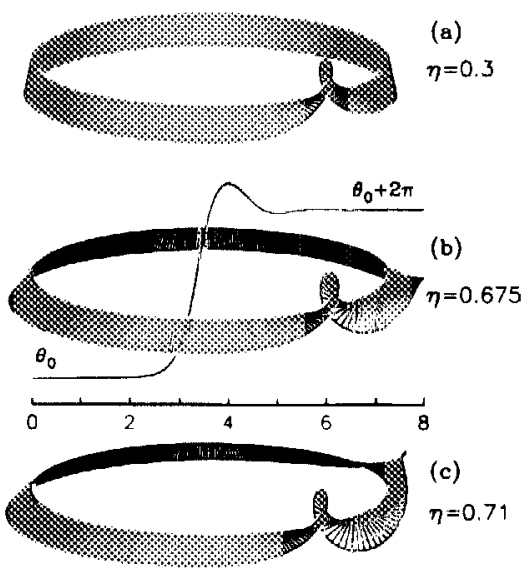

FIG. 2. Equivalent pendulum array for (a) $\eta=0.3$, (b) $\eta=0.675$, and (c) $\eta=0.71$. The inset in (b) shows the phase overshoot. $\alpha=0.01 ; \beta=0.02$.
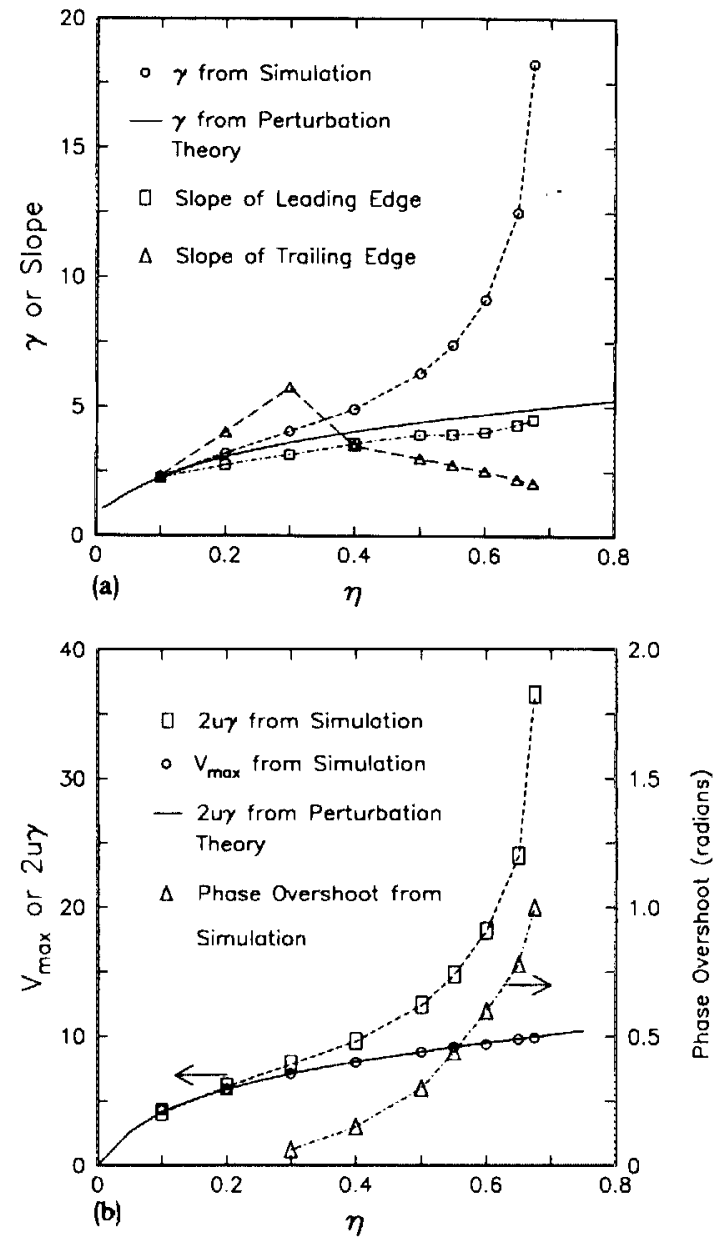

FIG. 3. Comparison for various quantities obtained from simulation and perturbation theory.

trailing edges of the soliton voltage waveform. According to Eq. (4) those slopes should numerically be equal to $\gamma(u)$, and is so observed for low $\eta$. Also in that regime $\gamma(u)$ from the simulation coincides with that obtained from perturbation theory, Eq. (2). A change in the slope of the trailing edge is seen to occur around $\eta=0.3$. This change occurs in connection with the appearance of the oscillations in the trailing edge. It is further observed from Fig. 3(a) that the Lorentz factor obtained from the simulations starts to deviate from that obtained by perturbation theory at about the same value of the bias current. Further, whereas the Lorentz factor, $\gamma(u)$, obtained from perturbation theory stays finite as $\eta$ is increased, we obtained $u \rightarrow 1, \gamma(u) \rightarrow \infty$ as $\eta \rightarrow \eta_{\text {sw }}$, where $\eta_{\mathrm{sw}}$ is the value of $\eta$ corresponding to switching.

In Fig. 3(b) is shown the phase overshoot at the trailing edge of the soliton. Note that this quantity diverges as $\eta \rightarrow \eta_{\text {sw }}$. Finally Fig. 3(b) shows the quantity $2 u \gamma(u)$ obtained from perturbation theory, Eq. (3), and from simulations, together with the maximum voltage obtained from the simulations. We note here that although $2 u \gamma(u)$ from perturbation theory is incorrect, the maximum voltage as obtained from Eqs. (2) and (4) is correct. In retrospect the maximum voltage may then be obtained with good precision from 


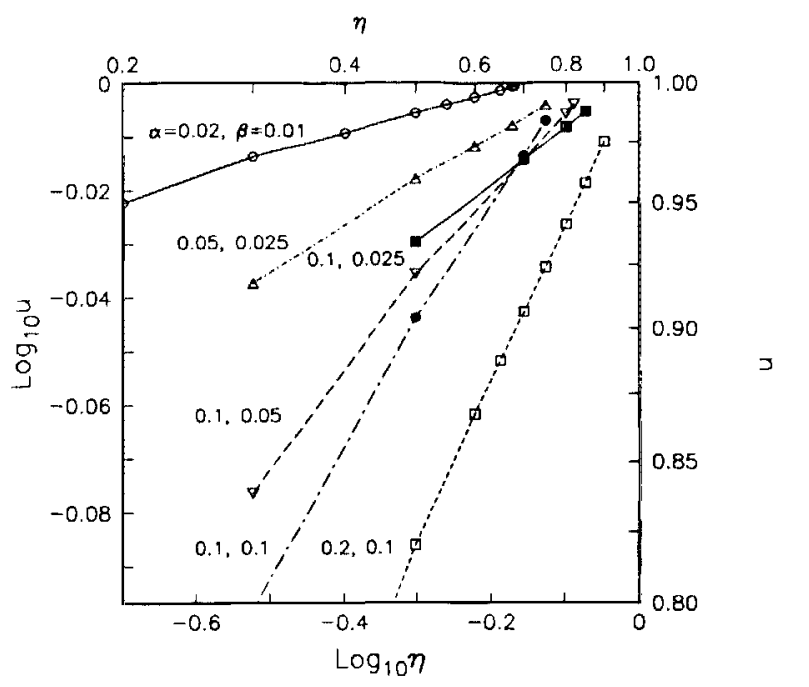

FIG. 4. $\log -\log$ plot of $u$ vs $\eta$ for high $\eta$. The damping parameters $\alpha, \beta$ are indicated in the figure.

$$
\begin{array}{ll}
V_{\max }=\frac{\pi \eta}{2(\alpha+\beta / 3)} & \eta \rightarrow 0 \\
V_{\max }=2(3 \pi \eta / 4 \beta)^{1 / 3} & \eta \rightarrow 1
\end{array}
$$

Figure 4 shows the results of the numerical simulations in a $\log u$ vs $\log \eta$ plot for various values of $\alpha$ and $\beta$. In such a plot it has been thought, based on analysis with $\beta=0$ that $u$ and $\eta$ would approach unity together. We note from Fig. 4 that $u \rightarrow 1$ for values of $\eta$ less than 1 , depending on the values of the damping parameters. We also find that in all the cases investigated, the junction is never stable when $u$ exceeds 1 , in contradiction to the results in Ref. 9. Our findings about $\eta_{\mathrm{sw}}$ are the following: with the ratio of $\beta / \alpha$ kept constant, $\eta_{\mathrm{sw}}$ increases with increasing damping level from 0.7 at $\alpha \beta=0.01,0.02$ to 0.92 at $\alpha \beta=0.1,0.2$. For a fixed $\alpha$ we find that $\eta_{\mathrm{sw}}$ decreases with increased $\beta$ losses. From Fig. 4 we may obtain an approximate empirical relation for the upper part of the $\eta-u$ curve which is not described by perturbation theory. We express it as a power law

$$
u \cong\left[1+0.044(\beta / \alpha)^{1.695}\right] \eta^{\alpha+\beta}
$$

Thus $u$ does not approach 1 asymptotically, but intersects the $u=1$ line at a finite slope, and with apparently a power law dependence on $\eta$.

A final point should be mentioned here. Following Marcus and Imry ${ }^{10}$ one may transform Eq. (1) to an ordinary differential equation of third order by assuming a travelingwave solution. The coefficient to the third order term then becomes $\beta u \gamma^{3}(u)$. We have found that simulations on this ordinary differential equation reproduce our simulations for low bias, but may lead to chaos for high bias, a result which is not found for Eq. (1) with periodic boundary conditions.

Stimulating discussions with B. Dueholm and M. Büttiker are acknowledged.

'T. Nagatsuma, K. Enpuku, F. Irie, and K. Yoshida, J. Appl. Phys. 54, 3302 (1983)

${ }^{2}$ T. V. Rajeevakumar, Appl. Phys. Lett. 39, 439 (1981).

${ }^{3}$ B. J. van Zeghbroeck, IEEE Trans. Magn. MAG-21, 916 ( 1985 ).

${ }^{4}$ A. Davidson, B. Dueholm, B. Kryger, and N. F. Pedersen, Phys. Rev. Lett. 55, 2059 (1985).

${ }^{5}$ D. W. McLaughlin and A. C. Scott, Phys. Rev. A 18, 1652 (1978).

'A. Davidson and N. F. Pedersen, Appl. Phys. Lett 44, 465 (1984).

'M. Büttiker and R. Landauer, Nonlinear Phenomena at Phase Transitions and Instabilities (Plenum, New York, 1982), p. 111; S. E. Burkov and A.

E. Lifsic, Wave Motion 5, 197 (1983).

${ }^{k}$ K. Nakajima, Y. Sawada, and Y. Onodera, J. Appl. Phys. 46, 5272 (1975).

${ }^{9}$ K. Nakajima, Y. Onodera, T. Nakamura, and R. Sato, J. Appl. Phys. 45, 4095 ( 1974).

10P. M. Marcus and Y. Imry, Solid State Commun. 33, 345 (1981). 